\title{
DE LA MUJER A LA COMPAÑNRA. PRESENCIA FEMENINA EN LAS MEMORIAS DE GUERRA ITALIANAS DE LOS AÑOS 30 Y 40 DEL SIGLO PASADO
}

\author{
FROM THE WOMAN TO THE PARTNER. FEMALE PRESENCE IN MEMOIRS OF \\ ITALIAN WARS IN 30S AND 40S OF LAST CENTURY
}

Manuel Gil Rovira

Universidad de Salamanca

\section{Resumen:}

A lo largo de la Historia, muchos documentos dan una prueba fehaciente de la realidad que se describe en sus páginas. Algunos de estos escritos, como las memorias, muestran el testimonio de personas que formaron parte en conflictos bélicos y reflejan la sociedad de aquella época. Este artículo pretende esbozar la imagen de la mujer en los conflictos que tuvieron lugar en la primera mitad del siglo XIX en Italia.

\section{Palabras claves:}

Máscara, guerra, mujer.

\section{Abstract:}

Throughout the History, many documents give reliable signs of the reality which is described on its pages. Some of these writings, such as the memoirs, show the testimony of people who took part in military conflicts and reflect the society in that time. This article aims to outline female image in conflicts which took place in the first half of the 19th century.

\section{KEY WORD:}

Goldoni, woman, theatre. 
La máscara es, como sabemos todos, en ese teatro clásico en el que sigue cimentada nuestra cultura, esa evidencia de los topoi, esa evidencia de los tópicos, esa evidencia sublimada de las relaciones humanas que demuestran el colectivo; esa evidencia sublimada del constantre vivir y del vivir constante que enquista o topifica, o simplemente convierte en habituales y cotidianas las relaciones humanas. La máscara es ese elemento de representación que resume y encarna el mito, en tanto que este se convierte en el discurso localizado y temporalizado fuera del espacio histórico con el que se devela lo fundamental y recurrente en el vivir humano de todos los días, en lo sancionado como habitual en la relación de los seres humanos con los seres humanos, de los seres humanos con la naturaleza. La máscara, tantas veces dotada de la significación de lo mágico, lo sobrenatural, lo "obligatoriamente" atendible, se reconduce en nuestra cultura occidental, en ese elemento de representación que concentra los rasgos arquetípicos de lo que la realidad ha dado, no sé si por bueno o simplemente por "real". Es por tanto símbolo y signo de lo real, de lo real dialógico, como no puede ser de otro modo en el espacio teatral, de lo real construido desde las relaciones socialmente admitidas o reproducidas. En la base de la utilización de la máscara está esa realidad que se construye desde la relación social, el colectivo, y que se ha dado por aceptada. En la base de la utilización de la máscara están desde la transmisión de esas estructuras sociales dadas como buenas, hasta la puesta en evidencia de éstas como una realidad tangible, injusta y, por tanto, carente de otra justificación que no sea la repetición del modelo que sólo encuentra en su propia existencia y recurrencia la razón de ser; que, puesto que sólo se explica desde discursos y modelos tautológicos, es susceptible de ser transcendida, traspasada, cambiada.

La máscara escrita, puede, por tanto, querer comunicar arquetipos y acabar así teniendo nombres codificados, encarnando personajes que definen qué entorno real que representan con su presencia todo lo que podría caber en un largo tratado de sociología. Desde Aristófanes, a la Commedia dell'arte, al Concierto de S. Ovidio, a Filumena Marturano, todas las máscaras, quieran representarse en cuero curtido o con la piel y el propio rostro humano, son, como así en parte los mitos, la reunión de una serie de características que resumen un tipo en relación con el entorno. En definitiva: la máscara, sublimación de la realidad.

En esa realidad subyacente, en lo que a fin de cuentas es máscara si estamos hablando de texto escrito, es en la que quiero centrar mi análisis. Y para ello me voy a detener de nuevo en una escritura, la escritura memorialística y de guerra, en la que, por sus características, el personaje femenino aparece codificado desde una escritura masculina. Aparece casi tipificado y, aunque las soluciones y posturas de los autores no serán plenamente coincidentes, lo que manda en la contrucción del retrato es la propia realidad, si por ésta entendemos, y así lo entendemos, no sólo la física presencia, que provocará variaciones en la visión de la persona, personaje mujer, sino la misma visión y la estructura social convivida que la informa. Voy a centrar, por tanto la intervención, en unos textos cuyos personajes son reales, reales con sus enteros nombres o con sus alias de guerra que, en cualquier caso, buscarán ser traducidos, sea en el corpus del texto sea en nota, con los nombres de pila que los identifican. Son un tipo de textos que, como ya he señalado en otros artículos sobre ellos, hunden su concepción en los datos recopilados de un diario de guerra sobre los que se rescriben unas memorias: la narración de lo anotado en el diario. Textos, en defintiva, que cuando rezan Fernanda, Carmen, Sandra (Onorina/Nori Brambilla) etc... están refiriéndose a esos personajes históricos datables y localizables, reales.

Evidentemente, estas memorias aparecerán más o menos noveladas y, en cualquier caso, nunca la escritura, por mucho que pretenda ser la transcripción de un diario, es un acta pura y notarial de la realidad. La realidad marca y codifica al personaje en gran medida, pero estos personajes femeninos codificados y clasificados van a ocupar un espacio concreto en la escritura, van a adquirir una importancia relativa en el conjunto de la narración. Como veremos, van a constituirse en símbolos de un conjunto con el que se quiere representar, y con el que, en cualquier caso, se lee e interpreta la realidad circundante. En definitiva máscaras.

Decía que desde la narración masculina y desde la realidad que también lo es, se iban a constituir los textos. Pero también decía que en esos textos que narraban acción, el desarrollo de la presencia femenina iba a ser diametralmente, no diré opuesto (lo impone la realidad narrada) pero sí distinto. Empezaré por la memoria actualizada de Tofoni, para centrarme finalmente en el desarrollo de la máscara mujer en lo escrito por Pesce. No desaparece en ninguno de los dos el "catálogo", los roles de la mujer encontrable, encontrada, en un tiempo, en el tiempo, en un conflicto bélico, en una guerra. Lo que en definitiva para algunos hombres de principios del siglo pasado, no constituye sino la representación al límite de las relaciones humanas. Pero en cualquier caso, la gradación, la importancia y la manera de narrar esas presencias, esas máscaras, van a ir desde algo parecido a melodrama, hasta algo parecido al realismo, con lo que ello conlleva de trasmisión de la idea o manera de representar la máscara mujer. Una será estática -aún levantando acta de la realidad-; la otra, tempranamente progresiva, acompañando esa misma realidad. La memoria de Ennio Tofoni, Il lungo cammino nella sierra, es clara. Sus mujeres -y perdónenme el posesivo, pero en ambos casos se adivina así, pues en ambos es un yo imperante- abarcan cuanto esperable, cuanto conocido en narraciones asimilables. Abarcan a la vieja campesina, que es madre de combatientes en el bando republicano. Abarcan a la miliciana internacionalista, de la que sólo se conoce el alias, y caída en combate. Abarcan a la miliciana compañera de lucha y de "sus labores" que en todo 
tiempo cuida del batallón, también caída en combate. Y abarcan a la enfermera del otro

bando, el amor de otrora, reencontrado.

- Sei sola, 'abuela' (nonna), gli chiese Enrico Belmonte.

- Si señor.

- Ti confido un segreto. Noi non siamo "los nacionales" ma "los rojos", come tu li chiami, siamo soldati della Repubblica.

La contadina congiunge le mani in atto di preghiera supplice e mormora:

- "Dios mio,", i miei figli sono con voi, ma non posso dirlo a nessuno. Mi fucilerebbero, come hanno fatto alla mia povera vicina.

- Ci siamo sperduti ed è per questo che ci troviamo in questi paraggi, fuori strada.

La brava donna continua a fare segno di stupore e a ripetere - "Dios mio, Dios mio".- Poi, guardandosi intorno con aria circospetta e trepida e con poche parole sussurra:

- Per carità, partite! Ripartite subito! Lasciate questi luoghi. La pattuglia "de los rebeldes" potrebbe ripassare da un momento all'altro. Un minuto che ritardate vi può essere fatale. Ho un nipotino che vi indicherà la strada accompagandovi. (Tofoni 1971: 73)

El personaje está en la narración. Es el entorno trágico y real, y son los clásicos demostrándonos cómo somos capaces de repetirnos a nosotros mismos.

- CLITEMESTRA. -Varones de nuestra ciudad, prez de los argivos, ninguna vergüenza voy a sentir de deciros cómo amo a mi esposo. Con los años pierde la timidez el ser humano. No voy a contaros algo aprendido de otras personas, sino las penas de mi propia vida, mientras él estaba al pie de Ilio En primer lugar, que una mujer se quede en su casa, lejos de su hombre, es una terrible tragedia. Oye continuamente rumores malignos: apenas ha llegado uno cuando otro trae un sufrimiento más grave que el anterior, todos diciendo a gritos desgracias para una casa..." (Esquilo 1986: 407)

La mujer doliente y agazapada. La que quiere saber del marido o del hijo. La que intuye dolor. Me van a permitir, porque estamos en un año que son cien años, la Josefina Manresa, que ya no espera, y es ella quien va a visitar en la cárcel de Alicante a Miguel. Y me van a permitir, porque cuando escribo estas líneas se acaba de ir un hombre importante que ha sido mucha vida con su compañera que aquí queda con nosotros, la Josefina Sempere que tejía los jerséis y hacía las grandes ollas para llevárselas a la cárcel de Carabanchel a Marcelino Camacho y sus camaradas de reclusión.

$\mathrm{Ma}$, ecco, le sirene riprendono a ululare e la gente raggiunge i rifugi nel metrò. $\mathrm{C}$ troviamo vicino alla "Puerta del Sol". Da lontano giunge il rombo degli aeroplani. D'un tratto, a poca distanza, esplode un obice. È la artiglieria nemica che dal monte di Carabancel segnala agli aeroplani il punto da bombardare. E gli aeroplani ora sono sopra di noi mentre le batterie antiaeree repubblicane entrano in azione. $\mathrm{C}$ rifugiamo dove arriva il sordo tonfo delle esplosioni: i Caproni e gli Junkers solcano il cielo rombando: le donne stringono al seno i figli. A stento trattengono le lacrime. Cessa l'allarme: usciamo. Le donne corrono trascinando per mano i bimbi (...). (Pesce 2006: 48-49).

El subrayado, evidentemente, es mío. La cita de Esquilo acoge las dos narraciones. Podríamos haber utilizado la Lisistrata de Aristófanes. Podríamos haber tirado de toda la tradición clásica que quisiéramos. Lo cierto es que el personaje femenino civil a secas, si así podemos llamarlo, y el personaje femenino civil actante, comprometido en la acción, están en ese representarnos la realidad desde esos tiempos. Así, decía, la representacion de mujer, su máscara, se irá construyendo en ambos autores, nuestros memorialistas, de manera distinta. Se deja entrever en las dos citas que he puesto como ejemplo. Ennio Tofoni, Bernardo en la narración y en la guerra, el que después será tenor reconocido en el ambiente del bel canto en Italia, narra las muertes de las mujeres comunes (como también Pesce). Forman parte del discurso de la normalidad, de cuanto sucede:

Intanto, Belmonte, accompagnato da due miliziani, si avviava verso il posto di soccorso passando di fronte una casa diroccata in cui aveva perso la vita la donna del villaggio. (Tofoni 1971: 127)

Sus mujeres, las de su narración, son también la miliciana, a la que se le dedica un capítulo entero en el que se llora su muerte. Ningna de las muertes requiere tanta atención en el texto como la de la miliciana, la de Carmen. Carmen es...:

Le batterie nemiche martellavano giorno e notte tardienta (...)

-Adesso il tiro si avvicina (...)

Che vuol dire andiamo? Andiamo dove? Meglio quil

-Avete paura -ribatte Carmen, che era intenta a preparare il caffé, con sorriso nervoso. (Tofoni 1971: 71)

Carmen es la compañera que prepara el café. Es la mujer miliciana y, puesto que mujer, la que más tiempo ocupa en la narración cuando se intuye que puede habe muerto; hasta saber y comprobar que ha muerto. Hasta saber que su cadáver está entre los escombros. El sentimiento de culpa invade como nunca a los hombres del batallón. Quien ha caído es Carmen, la miliciana mujer de la que se tiene la sensación de que había de ser protegida.

Nella nausea di quel sentore denso, il soldati pervennero al punto giusto, per scoprire finalmente il cadavere di Carmen gia in stato di decomposizione. La macabra coperta mise in evidenza l'atroce verità Carato di denosta alle ferite, seppur gravi, provocate dalle macerie, forse poteva verire seguito Carmen era stata vittima degli stessi uomini che volevano salvarla, a causa di un loro fatale errore di orientamento. E fu prima d'iniziare i lavori per rimuovere il cadavere che ebbero l'amara e raccapricciante sorpresa di scoprire la tragica verità. (...) La miliziana era piegata ed era ancora in vita e non fu difficile ricostruire l'episodio. Quando lei gemeva ed era ancora in vita, ad un tratto il suo corpo si trovo sotto il peso delle stesse persone che la cercavano; e per colmo di sventura, uno dei soccorritori piombò a caso picozza, la cui punta affondò sino a raggiungere la colonna vertebrale di Carmen, Uccidendola sul colpo. (Tofoni 1971: 127-128) 
Si los personajes que aparecen en la narración son casi necesarios, son casi lo que pasa, la mujer central, la que es la narración desde la mitad del relato hasta el final, la de "todo lo ocupas tú, todo lo ocupas", es Fernanda. Los necesarios volverán a aparecer. Pero quiero centrarme en Fernanda, el personaje que construye el melodrama, que es la vida de la narración y de quien narra, pero a la vez es una concepción, hasta heroica, de mujer, de la mujer que se entiende en el momento narrado $y$, todavía en el momento de la narración. Al fin y al cabo, la concepción de mujer que se intenta transcender en el siglo XX y que está aún vigente en el nuestro. Fernanda es el amor de otrora. La mujer que se casó con otro y la que, doliente de aquel, quiso cumplir con su memoria, que también era la patria.

$\mathrm{Si}$ andava verso la fine dell'estate. Un giorno, il 20 agosto, una giovane donna vestita di nero, si era indugiata verso il Commando militare di Roma, chiedendo di essere ricevuta dal Capo di Stato Maggiore.(...) -Vorrei rendermi utile, compiere in ogni modo il mio dovere di italiana. Mi accetti come crocerrossina. Mi sentirò moralmente più a posto. (...) Sposata al conte X nel 1934 la sua vita procedeva felice nella calda dolcezza di questa unione. Ma un giorno, della riserva, viene inviato in Abissinia, sul fronte della guerra. Poco dopo, cade in
un'imboscata. (Tofoni 1971: 45-46)

El personaje se sigue desarrollando hasta a llegar a ocupar el final de la narración. Belmonte cae herido y es atendido en un hospital del bando enemigo, il campo avverso. Allí se reencuentra con Fernanda, ahora la enfermera, la amada en otro momento.

\begin{abstract}
Quando Fernanda gli passava accanto, egli s'inchinava lievemente, in tutta dignità e pronunciava un rigoroso: "Buenos días señorita". Lei rispondeva con un educato cenno del capo I giorni trascorrevano così, monotoni, ma in vigile apprensione. Talvolta, il direttore Baros era risvegliato da improvvise ribellioni, irreprimibili, se pensava che "lei" amava un "nemico". "Amare un nemico! A che vale, allora una vit? A che serve odiare? L'amore, dunque, nel suo potere non ha senso. Il potere dell'amore è effimero e vano! Se si confondono amici e nemici nello stesso sentimento, quale è allora il valore di questa contrapposizione? (Tofoni 1971: 128-129)
\end{abstract}

El mismo planteamiento se repite con la muerte de Fernanda. Fernada es la mujer servicial, la pureza que "salva" la realidad del horror circundante.

Intanto nel campo avverso, Fernanda continuava la sua opera di conforto e di ausilio. La sua missione era ormai sostenuta da un sentimento puramente umanitario che si levava sopra tutte le predicazioni. (Tofoni 1971: 128)

Todo el capítulo 'Un soffio di gloria nell'eternità' va a ser la narración del cómo y el por qué se llega a la muerte de Fernanda; va a ser la narración del final de Fernanda como ejemplo de humanidad, sensibilidad, pureza si se quiere, parafraseando, de espíritu. Es toda la carga de melodrama de un texto operístico, no olvidemos que Tofoni era tenor. El desarrollo de todo el capítulo se centra alrededor de los dos personajes principales, Belmonte y Fernanda, que lo son a la vez de la entera obra. Son dos personajes en la escena de un campo de batalla que humanizan la narración desbordándola de sentimientos y pasiones trágicas. Es el combatiente republicano italiano Tofoni, y la enfermera en el bando nacional Fernanda. El campo de batalla es una ensenada de muertos. Una llamada desde el bando republicano a través de los altavoces convence a los ribelli de la necesidad de establecer una tregua secondo un piano di umanità para poder retirar a los caídos, muertos y heridos, de uno y otro bando. Así Belmonte / Tofoni sale con la ambulancia.

Mentre stava affaccendandosi per questo umano servizio, volge lo sguardo intorno la grande spianata e scorge, sui monticoli, un'infirmiera vestita di bianco. Trasale, sbarra gli occhi: non c'era dubbio era la snella figura di Fernanda.

(...) Ad un tratto l'aria è scossa da un sordo fragore. Tre caccia "nazionalisti" sorgono improvvisamente; e, non avvertiti in tempo, credono di trovarsi di fronte a un concretamento di forze repubblicane, soprattutto per il fatto che un enorme bandiera sventola nelle loro linee.

(...) Commilitoni e nemici si ritrovano insieme, si fanno posto uno accanto all'altro. (...) Un fatto tragicomico? No, un momento sublime di umana fraternità, che nasce dalla sostanza dall'essere e dal vivere.

(...) Belmonte non staccava gli occhi dal punto ove si trovava Fernanda, cercando di seguirla in tutti i suoi movimenti.

(...) Fernanda riprende la sua corsa. Le file repubblicane non si trovano più che a cento metri. È dunque lì che si indirizza. Ma è un attimo. La vede fermarsi, portare le mani al petto, vacillare, piegare di schianto a terra Enrico non resiste più. Balza dal suo riparo, e si precipita avanti.

(...) - Fernanda! Fernanda! Sono io, Enrico, ti porterò al mio campo... - Oh, Ennio, finalmente ti rivedo! Sono tanto felice!

(...)- Sai? Infine avevo compreso... volevo venire da te... Ma i "nostri" aviatori, -en (..)-Sai? Iire cò̀ Fernanda sorride con amarezza. I nostri amici aviatori hanno" giustizia" di tutto... Forse meglio così che aver sognato per nulla... I sogni sono nuvole... (Tofoni 1971: 150-152)

El capítulo, que es en realidad todo cuanto está alrededor de la muerte de Fernanda es en sí, y voluntariamente, toda una escena: la mejor delineada, por completa, de toda la narración; como lo es en el desarrollo de ésta el personaje de Fernanda, el más precisamente delineado, delimitado y presentado. Y si el marco melodramático decía que era completo, pues en él se describe esa máscara de mujer, en la escena deben callar los disparos y Belmonte debe cumplir la última voluntad de la amada. Fernanda le ha dicho que siga, que debe defender su esperanza, la de aquellos, todos, que creen en el mañana. Pero Fernanda tiene una familia y una vida en Italia y quiere quedar ante ellos y ante lo que aquella fue, como lo que había sido.

Finalmente Belmonte si ricorda delle calde e ultime volontà di Fernanda, e improvvisamente, come suscitato da un arcano richiamo della volontà, solleva il corpo di Fernanda e sorreggendolo nelle braccia, s'avvia lentamente, ma risoluto verso le linee nemiche. Il comandante medico Baros della sanità che aspettava i 
ritorno di tutti i suoi gregari e, di lei, (...) balzò fuori delle prime linee, facendo segno che non si sparasse. Furono inmediatamente impartiti ordini per evitare che si scatenasse una qualsiasi azione offensiva. (...) Gli uomini, grazie a Fernanda, avevano cessato di lottare, il suo olocausto risparmiava tante vite umane. (Tofon 1971: 154-55) (. I)'sotto l'ombra calma dei cipresi, ne saranno confortati dal pianto' 1971. 154-55) (...) sotto lombra calma dei cipresi, ne saranno confortati dal pianto (...) Fernanda e stata ricoperta da un gualcito lenzuolo. Il suo apostolato terren

ejo aquí de momento tras esta foscoliana cita la descripción de ese dibujo del personaje femenino en Ennio Tofoni. Ya había apuntado al principio cómo la caracterización de la máscara femenina, que responde a realidad y concepción, eran paralelas en la memorialística de Tofoni y de Pesce; cómo los personajes que relatan, que están en el relato, aparecen paralelos en ambas escrituras. Cuando hablaba de la mujer doliente, agazapada, introducía ya el paralelo entre lo escrito por ambos autores. Y planteando los textos de Pesce, ya en el ejemplo se vislumbraba, el calificativo de agazapada no tiene cabida. La mujer que configura nuestro piamontés es, al contrario, una mujer en acción, vestida de la acción del conjunto. La mujer que corre con el niño bajo las bombas que caen en la Puerta del Sol en Madrid, que veíamos en aquella cita, es la que convierte en plástica los dos párrafos anteriores.

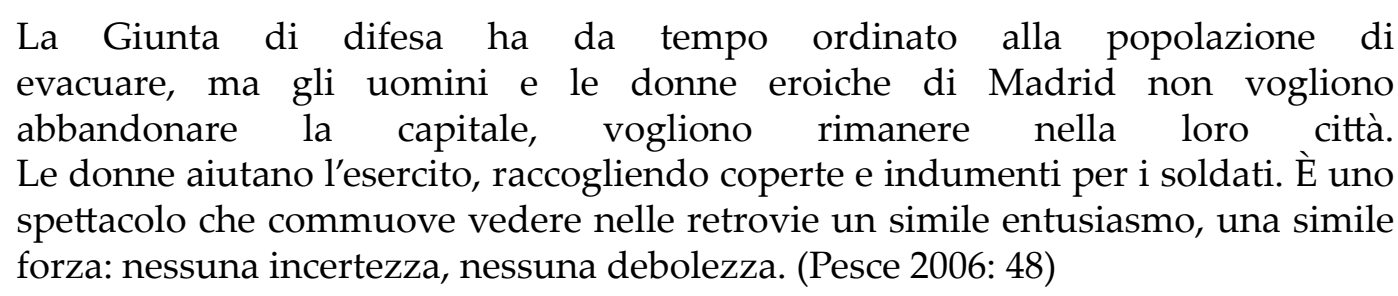

\section{ACCIÓN Y SUBSIDIARIEDAD EN MAYOR O MENOR MEDIDA VAN A SER LOS TRAZOS QUE DIBU-} JEN LA MÁSCARA EN NUESTRO PIAMONTÉS

El paralelo que quizá se presenta de manera más clara entre las dos escrituras, aunque sólo sea por la igualdad de rol y por el aspecto estructural en la narración, es el personaje de Carmen. Carmen es, al igual que Fernanda, un capítulo entero y, como aquélla, es enfermera, en este caso republicana. A Fernanda no se la presenta como enfermera 'nacional', sino como voluntaria venida en su viudedad y su bondad. A Carmen, sin embargo:

Faccio conoscenza con l'infemiera Carmen, una giovane ragazza spagnola. I suo genitori sono emigrati in Francia. E venuta volontaria in Spagna, ha frequentato il liceo a Montpellier e parla correttamente il francese. (Pesce 2006: 132)
Es, como Fernanda, la mujer que cuida de los enfermos, que se preocupa del estado, no sólo físico, sino de ánimo de los heridos. Pero lo es desde el doble espacio, el de la mujer enfermera y el de la mujer convencida de la acción.

Carmen è bella e molto buona, sa trovare per ognuno una parola dolce e umana, è affabile con tutti. Ogni mattina, avvolta nel suo camice bianco, viene a medicare tutti. La sera, passeggiando, faccio lunghe discussioni con Carmen alle quali assiste talvolta il tenente Giua. (Pesce 2006: 132)

La misma con la que unos párrafos, más adelante:

Ci sediamo sulla sponda del balcone, mi parla del suo paese, Montpellier. Si anima parlando del suo lavoro, della sua missione. Quando pronuncia la parola "missione" la guardo attentamente e scopro in lei la forza di volontà, la passion che essa mette nel soccorrere... (Pesce 2006: 134)

Carmen es el amor del momento del garibaldino sin nombre (sólo aparece una vez, y es al final, en toda la narración) de la que se querría oir la voz mientras se duerme: "je t'aime e tu" y de la que, pasado un tiempo se sabrá de su muerte.

Verso le 9,30, Carmen entra in corsia al seguito del medico: i nostri occhi si incontrano, la guardo con timidezza. Devo essere rosso in viso quando $\mathrm{mi}$ si avvicina per la solita medicazione - Perché sei confuso? Di che cosa ti vergogni, hai paura di me? Che garibaldino sei? (...) Non l'ho più rivista. È successo pochi mesi dopo. Ė rimasta uccisa in seguito ad un mitragliamento di aeroplani fascisti. (Pesce 2006: 133, 135)

Así, las dos máscaras femeninas más dibujadas en ambas narraciones son Fernanda y Carmen. Es cierto que hay una diferencia de presencia en la estructura. Si Fernanda es el segundo personaje en Tofoni, no es así en el caso de la Carmen de Pesce. La amada de un momento de Pesce es una pincelada concreta, si bien muy importante, de un retrato colectivo. En cualquier caso, y teniendo en cuenta este hecho, la diferencia en el dibujo de la realidad femenina en ambos autores masculinos parece intuirse. Si seguimos cogiendo el retrato en conjunto de la presencia mujer en la obra de Pesce, encontraremos en nuestro garibaldino un momento interesante de inflexión o de representación de realidad. Va a ser en una escena en Madrid en la que aparecen dos milicianas fuera de las escenas de combate. Dos milicianas en ese momento en la retaguardia, al igual que nuestro piamontés y sus camaradas que descansan en la ciudad. En las calles de Madrid, Gilfredo, otro garibaldino camarada de Pesce, encuentra a un viejo amigo español al que había conocido en París en 1932. El amigo les invita a comer en su casa con su familia y durante la comida se produce una escena que, en la narración, marca un poco la presencia femenina que introduce nuestro memorialista. 
Se intuye, en cualquier caso, una realidad de cosas de ese primer tercio del siglo XX en España, aunque después retrocediera, se reprodujera y durara todo el siglo.

Ogni tanto la madre, rivolgendo lo sguardo verso le ragazze, le rimprovera per essersi arruolate volontariamente nella milizia. Invano Cerbai e Storai, due garibaldini che sono con noi, cercano di prendere le loro difese. -Che c'entrate voi con la guerra? Io sono vecchia e le mie figlie mi abbandonano. La guerra la devono fare gli uomini, -ripete rivolgendosi a noi. - Tu sei una mama gara, ma piena di pregiudizi. Certe cose non le proi -Tu se una man cuo capire. E poi ti ricordi quante donne sono scese sulla strada a fianco degli uomini quando scoppio la rivolta nel luglio del '36?- rispondono le figlie. La più grande accarezza la madre e con un bacio cerca di avere la sua approvazione. (Pesce 2006: 28-29)

No es elección de Pesce, por tanto, o no lo es sólo, la manera de dibujar esa máscara. Es la máscara de la realidad la que le impone esa mujer / acción. Es la máscara de la realidad y, lógicamente, de lo que quiere contar. Si hay un personaje activo en la escritura de la Guerra de España, es el de Estella. Estella, alias de guerra de Teresa Noce, aparece en el texto de nuestro garibaldino, con su nombre real, salpicando distintos momentos de la narración, pero siempre visitando el frente. Aparece junto a otros dirigentes, Luigi Longo (su compañero), Giuliano Pajetta, Pacciardi. Pero aparece, sobre todo, como lo que es, la periodista en el frente, la que, como decía, conocemos firmando sus escritos como Estella en distintos periódicos y en publicaciones conjuntas durante la guerra.

PrimoMaggio:Festainternazionale deilavoratori.In accordo conle autorità cittadine organizziamo una grande manifestazione con sfilata. Con noi vi sono Longo, Nenni, Barontini, Pacciardi e altri ufficiali. Teresa Noce parla ai soldati e al popolo. Pacciardi consegna ai pionieri due bandiere offerte dalla brigata. (Pesce 2010: 95) Così Barontini, in una intervista concessa a Teresa Noce, spiega l'ardimento dei garibaldini del terzo Battaglione... (Pesce 2006:112)

Aparece como el indicio, que se sabe desde las trincheras de Quintanar de la República, del texto que está actuando para ellas:

A Quintanar sono arrivati i pacchi-dono inviati dall'emigrazione, soprattutto in seguito all'interessamento di Teresa Noce, e del giornale 'La voce degli italiani' (Pesce 2006: 142)

Si cuando hablaba de la escena de la comida familiar en Madrid, escena muy temprana, intentaba plasmar una especie de canon de descripción e idea de la realidad mujer en la narración, el primer nombre de mujer dirigente, o bien, la primera mujer nombrada, cuando ya han aparecido mujeres, niños y hombres anónimos recibiendo a los internacionalistas, es el de Pasionaria. En otra entrevista/memoria ha contado
Pesce cómo una de las cosas que lo trajo a España habían sido las palabras de Dolores en París en la primavera del '36. Así, en las primeras páginas de nuestra narración podemos leer:

Sul monumento di Narciso Monturiol, l'inventore del sottomarino spagnolo che fece il percorso Barcellona-Alicante nel 1869, erano arranpicati gruppi di ragazzi che salutavano con il loro piccolo pugno. Allineati di fronte alla stazione, ascoltammo 1 discorso di un rappresentante del comune. L'uomo aveva appena terminato con le parole della Pasionaria: 'Se la Spagna fosse abbattuta, dei torrenti di sangue inonderebbero il mondo immediatamente; lo spettro della guerra si leverebbe su tutti gli orizzonti', che noi rispondemmo di fare il nostro dovere. (Pesce 2006: 18)

Es desde esa mujer acción, desde esas palabras que siempre nos han traído al pensamiento los memorables versos de Vallejo, Niños del mundo / si España cae -digo, es un decir- / si cael (...) qué pronto en vuestro pecho el ruido anciano! / qué viejo vuestro 2 en el cuaderno. / (...) si no veis a nadie, si os asustan / los lápices sin punta, si la madre/ España cae -digo, es un decir- / salid, niños del mundo; id a buscarla!... (Vallejo 1983: 303-304), desde donde vamos a entender la mujer con nombres en Pesce.

No podemos cerrar el círculo de la mujer con nombre en Pesce sin hablar de Sandra. Cuando nuestro piamontés salió de España y tras haber pasado por su casa en la Grand' Comb', va a Italia, su país natal, donde es detenido y confinado. Al acabar el confinamiento se incorpora a la lucha antifascista. Todo ello está narrado en otras memorias: Senza tregua. La guerra dei GAP. Senza tregua sí que tiene dos personajes principales. Es la narración de la lucha en solitario del saboteador que así apoya organizado la lucha partisana desde dentro de las ciudades. Es la misión que le ha sido encomendada. De entre todos los personajes que van apareciendo incluido el de Dante di Nanni, Sandra es, en la segunda parte, el más importante, el que podríamos llegar a entender como el otro personaje principal, en una narración que sólo los tiene capítulo a capítulo. Sandra son muchas máscaras que acaban resumiéndose. Es el disfraz de la correo que pasa desapercibida mientras hace de unión entre los elementos de la célula. Es la cara escondida que vigila mientras se realiza la acción. Sandra es, en definitiva la compañera. El 12 de septiembre a las 17 horas, el dirigente, Visone (Pesce -en esta narración el protagonista sí que tiene nombre a través de sus alias-), tiene que acudir a una cita en Piazza Argentina que parece ser una trampa. La enviada es Sandra.

(...) Sandra si avvicina come se non lo conoscesse. Dirà la parola d'ordine con l'aria di chiedere una informazione e riprenderà subito a camminare se l'altro non rispondera con la contraparola. (...) Segnalato il pericolo, invece di allontanarsi di lei, la prende sottobraccio. E assurdo. Poi come ricordando improvvisamente regole della clandestinità, si allontana quasi di corsa Sunta alle spalle un (Pesce 1973: 217-218) 
Sandra ya no es la mujer, es la compañera que acaba de caer. El protagonista narrador, Visone, narra la espera, la intención y los planes para liberarla. Y, entre tanto el narrador cuenta lo que no ve ni le han contado. Nadie dice "tal ha oído", "hemos sabido". Hay una interrupción en la narración señalada, como otras veces, a través de un asterisco y, a través de no se sabe qué ojos:

La cella, nel carcere di Monza è buia e silenziosa. Dopo le luci abbaglianti delle lampade, dopo (...) Sandra si sente quasi protetta dall'oscurità e dal silenzio. (...) 'Dov'è Visone?' Ha risposto ostinatamente di no conoscerlo (...) 'Che ne diresti se arrestassimo la famiglia di questa signorina?' Nessuno risponde. (Pesce 1973: 220)

Sandra se ha convertido casi, sólo casi, en narradora. El narrador siempre es Pesce. Pero si algo puede dar idea de cuál es la idea que sobrevuela sobre estas memorias y su recepción por el lector en cuanto a ese compartir protagonismo, es el documental homónimo que Marco Pozzi hizo en el año 2004, basándose en este texto. La imagen constante es la de la pareja Visone (Giovanni Pesce) y Sandra (Nori/ Onorina Brambilla) hablando uno y otro alrededor de una mesa camilla. Aún así, el cartel que lo anunciaba era el de una foto de juventud tras la liberación en el que Sandra aparece cogiendo del brazo a Visone y un poco atrás, relegada.

La mujer doliente. La civil que sufre todas las guerras. La mujer protagonista, la que tiene nombre presente en la narración. Y ahí, la mujer narrada, vivida como una escena de melodrama o la vivida como un elemento en acción, como quien 'agarra' al niño para salvarlo de las bombas de otros. Todo el espectro mujer desde lo masculino narrante. Todo el espectro que va de la narración melodramática, en unas memorias, las de Tofoni, a la descripción realista, en unas memorias, las de Pesce. El protagonista, siempre masculino, es el narrador y sus memorias. Pero las máscaras de las mujeres, entendiendo siempre que son narradas, tienen los pómulos y hasta la acción de las manos distintas. Permítaseme esta metáfora para definir la diferencia. En una narración, la de Tofoni, las manos de la mujer se mueven pasivas. En la otra, las otras narraciones, las de Pesce, las manos de la mujer se mueven activas. Y en ambas el narrador es el hombre.

\section{REFERENCIAS BibLIOGRÁFICAS}

Alessandrini, L. et alii, Immagini nemiche. La guerra civile spagnola e le sue rappresentazioni 1936 1939, Bologna, Editrice Compositori, 1999.

Esquilo, Agamenón en Tragedias, trad. y notas B. Perea Morales, Madrid, Gredos, 1993.

Estella (Teresa Noce), “Presentazione”, en AA.VV. Garibaldini in Ispagna, Madrid, Diana-UGT, 1937
Giannantoni, F.-Paolucci, I., Giovanni Pesce "Visone"un comunista che ha fatto l'Italia, Varese, Edizioni Arterigere-Essezeta, 2005

Gil Rovira, M., “Oggi in Spagna, domani in Italia” en F. Saverio y R. Mª Grillo (eds.), La Spagna degli anni '30 di fronte all'Europa e all'America, Roma, Antonio Pellicani Editore, 2001.

----, "Memorialística garibaldina en la Guerra de España", en V. González Martín (ed.), La filología italiana ante el nuevo milenio, Salamanca, Ediciones Universidad de Salamanca, 2003, pp. 221-232.

---, “Los 'garibaldini' escriben España” en A. R. Celada (et alii), Las Brigadas Internacionales: 70 años de memoria histórica, Salamanca, Ediciones Amarú, 2007, pp. 183-189.

----, "Garibaldini e memoria scritta nella guerra di Spagna" en AA.VV., Tempo e memoria nella lingua e nella letteratura italiana, Associazione Internazionale Professori d'Italiano (en prensa).

Pesce, G., Senza tregua. La guerra dei GAP, Milano, Universale Economica Feltrinelli, 2005 (19671).

----, Un garibaldino in Spagna, Varese, Edizioni Essezeta-Arterigere, 2006 (Roma, Editori Riuniti, 19551).

----, Un garibaldino en la Guerra de España, introd., trad. y notas de M. Gil Rovira, Madrid, Atenea (en prensa)

Pozzi, M., Senza tregua, Roma, Sharada Film, 2004.

Tofoni, E., Il lungo cammino nella sierra, Milano, Nuova Tipolito Edizioni Lavoro, 1971 (Archivo Histórico del PCE).

Vallejo, C., Obra poética completa, Madrid, Alianza editorial, 1983. 